synthesis of the molecule to marketing approval, has more than doubled (from 6.5 to about 14 years) since 1964 . And development costs have skyrocketed: According to the Tufts Center for the Study of Drug Development, research-based companies' average expenditures to bring a new drug to the end of clinical testing were, in 1970, 1980, 1990 and 2003, \$138 million, $\$ 318$ million, $\$ 802$ million and $\$ 897$ million, respectively.

Moreover, whereas between 1970 and 2000 research-based companies' R\&D outlays soared from about $\$ 2$ billion to $\$ 30$ billion, the number of approvals of new drugs barely budged. (There are influences besides government regulation that partly account for the increased complexity and expense of clinical trials, and for the rising costs of drug development overall. For example, there may have been a subtle shift in corporate strategy: drug sponsors now attempt to amass data to demonstrate not only safety and efficacy as measured against a placebo or standard therapy, but also against certain competing products, to allow a claim of superiority after approval. However, an important factor is the obsessively risk-averse FDA continually raising the bar for approval, especially for innovative, high-tech products.)

The trend in cancer drug approvals is illustrative. Rothenberg et al. ${ }^{1}$ have analyzed trends in FDA approvals of drugs and indications (uses) and conclude, "When projected out over the 5-year period of 2000-2004, [the data predict] a 68\% reduction in new drug approvals and a $37 \%$ reduction in the number of claims approved for new oncologic indications, compared to the preceding 5-year period of 1995-1999."

Even with good intentions, FDA Commissioner Mark McClellan seems unlikely to make a dent in the agency's dismal performance. He confronts a 'corporate culture' of risk aversion and timidity, and there's so little in the pipeline-the number of marketing applications for new molecular entities filed with the FDA has been decreasing steadily since 1995-that even greater motivation and better management at the agency would have little substrate on which to work.

\section{Henry I. Miller}

The Hoover Institution, Stanford

University, Stanford, CA 94305-6010,

USA

e-mail: miller@hoover.stanford.edu

1. Rothenberg, M.L., Carbone, D.P. \& Johnson, D.H. Nat. Rev. Cancer 3, 303-309 (2003).
Alan Dove responds:

Miller claims my article contains

"misapprehensions and misstatements," with the implication that I relied too heavily on FDA sources. I strongly disagree with his assessment. The data on clinical trials and approval times are open to interpretation. The researchers who compiled the data favor the view that the FDA has become much more efficient, but a variety of internal and external forces have conspired to lengthen corporate-sponsored clinical trials. In an era of intense competition and litigation, the FDA is not the only organization that is risk-averse. Background research for this article was extensive, and included interviews with a range of experts, most of them outside the FDA. Conclusions about the agency's performance relied on statements by drug industry sources and independent observers, and comparison with analogous organizations overseas.

\title{
A TIP on malaria (genomics)
}

\section{To the editor:}

In the March issue, Fiscella et al. (Nat. Biotechnol. 21, 302-307, 2003) identify a novel human T-cell immunomodulatory protein (TIP). Their study has direct relevance for massive BLAST searches that we have carried out at the

DNA level to identify genes responsible for centriolar mitosis by fishing out genes present in animals and unicellular flagellates and not in other types of organisms.

Among such genes we have found in the Plasmodium falciparum genome, which mostly encode proteins participating in construction of the flagella, there is also a homolog of TIP, annotated a 'hypothetical protein' Q8I3H7. Indeed, when a Smith-Watermann search is carried out using TIP as a query, this Plasmodium protein is found (with an expect value of 1.06E-108) as the only non-animal protein homologous to TIP. The two proteins share $29.6 \%$ identical residues along the whole length. Analysis with SMART ${ }^{1}$ (http://smart.embl-

heidelberg.de/) reveals that the TIP and Q8I3H7 also have a similar structure, both containing transmembrane domains.

Given the relatively low homology between the two proteins, as well as low homology between Plasmodium protein and its homolog from the natural mosquito vector (Anopheles gambiae and Plasmodium proteins share $25 \%$ identical residues), the possibility of recent horizontal gene transfer appears unlikely.

In this light, there are two possible explanations for the presence of a TIP homolog in the Plasmodium genome. Q8I3H7 may be an old secretory protein necessary for the Plasmodium lifecycle, further adapted to T-cell regulation. Alternatively, a very interesting possibility is that the TIP homolog is involved in P. falciparum's parasitic lifestyle and its ability to immunomodulate the host organism. T cells have been shown to play a major role in early stages in protective immunity against malaria parasites ${ }^{2}$.

Analogous to

the protective effect of TIP protein in graft-versus-host disease described by Fiscella et al., Q8I3H7 may protect the parasite against attack by the host immune system.

Szymon Kaczanowski \& Piotr Zielenkiewicz

Institute of Biochemistry and Biophysics,

Polish Academy of Sciences,

Pawinskiego 5a, 02-106 Warszawa,

Poland

e-mail: (piotr@ibb.waw.pl)

1. Schultz, J., Milpetz, F., Bork, P. \& Ponting, C.P. Proc. Natl. Acad. Sci. USA 95, 5857-5864 (1998)

2. Tsuji, M. \& Zavala, F. Trends Parasitol. 19, 88-93 (2003). 